\title{
IMPORTANCIA DE LA ALTITUD EN LA FISIOPATOLOGÍA DEL COVID-19
}

\section{IMPORTANCE OF ALTITUDE IN THE PATHOPHYSIOLOGY OF COVID -19}

\section{ALTURA Y COVID 19}

Manuel Montoya Lizárraga 1,2

Citar como: Montoya Manuel

1 Universidad Nacional de San Antonio Abad del Cusco. Cusco, Perú.

2 Hospital Regional del Cusco, Perú

a Médico Especialista en infectología

ORCID: https://orcid.org/0000-0002-0556-0299

Anahí K. Cardona Rivero 1

Citar como: Cardona-Rivero AK

1 Universidad Nacional de San Antonio Abad del Cusco. Cusco, Perú.

c Bioquímico farmacéutico.

ORCID: https://orcid.org/0000-0001-6397-9162

\section{Contribuciones de los autores:}

Manuel Andrés Montoya Lizárraga y Anahí Karina Cardona Rivero realizaron el análisis y elaboración del artículo de revisión.

6. Financiamiento: autofinanciado

\section{Conflictos de interés:}

Yo Manuel Andrés Montoya Lizárraga declaro no tener conflictos de interés, no haber recibido pago alguno de ninguna institución, tampoco tengo ningún plan de patente, ni otras relaciones o actividades que puedan afectar la objetividad del manuscrito; según lo señalado en la Declaración Jurada y el formulario de conflictos de interés (COI).

Yo Anahí Karina Cardona Rivero, declaro no tener conflictos de interés, no haber recibido pago alguno de ninguna institución, tampoco tengo ningún plan de patente, ni otras relaciones o actividades que puedan afectar la objetividad del manuscrito; según lo señalado en la Declaración Jurada y el formulario de conflictos de interés (COI).

\section{Correspondencia:}

Nombres y Apellidos: Manuel Andrés Montoya Lizárraga.

Dirección: Urb. Versalles f-6 San Jerónimo Cusco.

Email: manuel.montoya@unsaac.edu.pe

SITUA. 2020; 23(2): 1-19

DOI: https://doi.org/10.51343/si.v23i2.310
Fecha de recepción: 15 de julio del 2020

Fecha de aceptación: 10 de diciembre del 2020 


\section{IMPORTANCIA DE LA ALTITUD EN LA FISIOPATOLOGÍA DEL COVID-19}

\section{RESUMEN}

La pandemia de COVID 19 inicia en diciembre del 2019 en China, se extendió a casi todos los países del orbe con celeridad, poco antes vista por la capacidad de transmisión y el grave impacto en la salud pública, con gran cantidad de infectados y letalidad elevada. Tiene un comportamiento diferente con relación a la altitud de las zonas geográficas donde se presenta y es consistente en países que inicialmente fueron afectados y se encuentran en resolución o estabilización de la enfermedad como China o en desarrollo inicial o crecimiento exponencial como en América Latina. Teniendo un comportamiento menos agresivo y menor mortalidad, estos disminuyen a medida que la altitud es mayor de los 2500 m.s.n.m.

Dentro de la adaptación de estas poblaciones sometidas crónicamente a hipoxia hipobárica, está la alteración del Sistema Renina Angiotensina (SRA), determinada por factores genéticos. Coincidentemente dentro de la fisiopatología del COVID-19 el principal sitio de unión a la proteína $\mathrm{S}$ espícula viral es el receptor del ECA2, por lo que su disminución o ausencia disminuiría su virulencia.

Estas posibilidades son examinadas, recordando las condiciones del habitante de altura, las características genéticas y su relación con el SRA, la implicancia en la enfermedad y la evidencia de casos respecto a las principales áreas geográficas de altura.

Esta es una contribución en la evaluación de la relación del COVID19 y la altitud, que en influirá en la forma del enfoque de la enfermedad, manejo social y salud de los afectados.

Palabras clave: COVID-19; Altitud; Hipoxia Hipobárica (fuente: DeCS BIREME).

\section{SUMMARY}

The COVID 19 pandemic begins in December 2019 in China, spreading to almost all orb countries quickly, shortly before seen by transmission capacity and the severe impact on public health, with large numbers of infected and high lethality. It has a different behavior in relation to the altitude of the geographical areas where it occurs and is consisting of countries that were initially affected and are in resolution or stabilization of the disease such as China or in initial development or exponential growth as in Latin America. Having less aggressive behavior and lower mortality, these decrease as the altitude is greater than 2500 m.s.n.m. Within the adaptation of these populations chronically subjected to hypobaric hypoxia, is the alteration of the Renina Angiotensin System (SRA), determined by genetic factors. Coincidentally within the pathophysiology of COVID-19 the main site of binding to the viral spicular S protein is the receptor of ECA2, so its decrease or absence would decrease its virulence.

These possibilities are examined, recalling the conditions of the height dweller, the genetic characteristics and their relationship with the SRA, the implication in the disease and the evidence of cases regarding the main geographical areas of height.

This is a contribution in the evaluating of the relationship between coVID19 and the altitude, which will influence the shape of the disease approach, social management and health of those affected. Key words: COVID-19; altitude, Hypobaric Hypoxia (source: MeSH NLM). 


\section{INTRODUCCIÓN}

Es conocida la fisiología del poblador de altura donde la Hipoxia Hipobárica $(\mathrm{HH})$ a gran altitud da como desarrollo mecanismos compensatorios como: la saturación de oxígeno arterial reducida, perfusión de órganos con sangre hipoxemia e hipoxia directa de tejidos pulmonares. Las adaptaciones pulmonares en las células de las arteriolas pulmonares debido a $\mathrm{HH}$ es la base de los mecanismos fisiopatológicos de la altitud (1). También se observa una adaptación del sistema renina angiotensina (SRA) teniendo representación genética con carácter fenotípica cuando se encentran expuestos a HH. y de la adaptación a la altura crónica y la patología de enfermedad aguda (1) (2)

La presente pandemia de COVID 19, se ha mostrado que tiene una gran transmisibilidad con su secuela de morbimortalidad que se encuentran con manifestaciones diferentes en poblaciones, mostrando mayor virulencia en unas y en otras, así como se evidencia que los sitios de adherencia del SARS-COV2, son la proteína $S$ o espícula del virus con el receptor que principalmente es La enzima convertidora de angiotensina 2 (ECA2) que se localiza en la superficie de celular de varios órganos en especial de las vías áreas superiores e inferiores desencadenado el proceso fisiopatológico (3)(4)(5).

Al observar que la presencia y agresividad del COVID 19 en poblaciones de altura es menor, buscamos las posibles causas que explicarían estos hechos. Enfocándonos en la alteración del SRA, con su expresión de la ECA y las características genéticas en estas estructuras del habitante de altura. Lo cual podría incidir en algunas formas de enfocar la pandemia en estas poblaciones.

\section{ADAPTACIÓN A LA ALTURA}

Hipoxia Hipobarica $(\mathrm{HH})$ se conceptúa como la disminución de la de Presión parcial de oxígeno en el aire inspirado, pero conserva la proporción del $21 \%$, en la medida que se asciende a mayor altura donde la presión barométrica va disminuyendo de $760 \mathrm{mmHg}$ a nivel del mar, a menos. Por lo que a mayor atura menor Presión barométrica y menor Presión de oxígeno atmosférico (tabla 1) (1) (6).

Tabla 1. Correlación entre altitud, presión barométrica, presión parcial de oxígeno atmosférico (ambiente seco) inspirado (con vapor de agua) y presión parcial de oxígeno arterial

\begin{tabular}{|c|c|c|c|c|}
\hline Altitud & $\begin{array}{c}\text { Presión } \\
\text { Barométrica }\end{array}$ & $\begin{array}{c}\text { Presión parcial } \\
\text { de Oxigeno } \mathbf{P O}_{\mathbf{2}}\end{array}$ & $\begin{array}{c}\text { Presión Parcial } \\
\mathbf{O}_{\mathbf{2}} \text { inspirada }\end{array}$ & $\begin{array}{c}\text { Presión Parcial } \\
\mathbf{O}_{\mathbf{2}} \text { arterial } \\
\text { (PaO2) }\end{array}$ \\
\hline Metros & $\mathbf{m m H g}$ & $\mathbf{m m H g}$ & $\mathbf{m m H g}$ & $\mathbf{m m H g}$ \\
\hline $\mathbf{0}$ & 760 & 159 & 149.3 & 99.7 \\
\hline $\mathbf{5 0 0}$ & 716.0 & 150.0 & 140.0 & 90.5 \\
\hline $\mathbf{1 5 0 0}$ & 634.3 & 132.9 & 123 & 73.3 \\
\hline $\mathbf{2 5 0 0}$ & 560.2 & 117.4 & 107.5 & 57.7 \\
\hline $\mathbf{3 5 0 0}$ & 493.4 & 103.4 & 93.5 & 42.7 \\
\hline $\mathbf{4 5 0 0}$ & 433.2 & 90.7 & 80.9 & 31.1 \\
\hline $\mathbf{5 5 0 0}$ & 405.4 & 84.9 & 75.1 & 25.2 \\
\hline
\end{tabular}




\begin{tabular}{|l|l|l|l|l|}
\hline $\mathbf{6 5 0 0}$ & 330.6 & 69.3 & 59.4 & 9.4 \\
\hline $\mathbf{7 5 0 0}$ & 287.3 & 60.2 & 50.2 & 0.4 \\
\hline
\end{tabular}

Fuente: Adaptado de Avellanas. 2018. (1)

Las poblaciones que han vivido en $\mathrm{HH}$ durante miles de años a grandes alturas por más de 2500 msnm se han adaptado evolutivamente a este estrés ambiental. Desarrollando respuestas fisiológicas adaptativas y desadaptativas que incluyen alteraciones en vías como la detección de oxígeno, sensibilidad a la hipoxia disminuida, bomba de sodio potasio, activación de canales de calcio, equilibrio redox y el sistema renina-angiotensina-aldosterona (7)

En la personas que ascienden a la altura mayores a 2,500 msnm, se producen cuadros clínicos relacionados con susceptibilidades genéticas y respuestas no homeostáticas que predispone a enfermedades de mal de altura o de montaña o "soroche, como son la salida del líquidos del intravascular a los tejidos, edemas, (8). En cuanto a la, secreción de aldosterona esta disminuida por inhibición que produce la hipoxia sobre las células glomerulares del riñón (9)

Los mecanismos de adaptación a la altura son: Disminución del volumen extravascular, aumento de la excreción renal del Sodio, esto en relación con la baja concentración de renina, aumento del aclaramiento renal y mayor flujo urinario. Motivando la baja de angiotensina II.(10) Con la altura si bien la presión de oxígeno es baja, la mitocondria no para su acción de producción energética (11) .

En normoxia, el sistema RAS está regulado por el equilibrio dinámico entre la expresión de ACE1 y ACE2. Sin embargo, bajo hipoxia crónica ( $\mathrm{O}_{2} 2 \%$ durante 12 días) ACE1 está regulado por el factor inducible por hipoxia 1 (HIF-1) en las células del músculo liso de la arteria pulmonar humana (hPASMC), mientras que la expresión de ACE2 disminuye notablemente (12).

HIF-1 como regulador maestro en caso de hipoxia, regula la homeostasis en esa condición y frente a la $\mathrm{HH}$. Se puede hallar en muchos tejidos. Se degrada con la normoxia y crece exponencialmente con la hipoxia (13). (14).

\section{EL SISTEMA RENINA-ANGIOTENSINA (SRA)}

Compuesto por la enzima renina que limita la velocidad de activación y un principal impulsor de péptidos, la angiotensina II (Ang II) que actúa a través de su receptor de subtipo-1 (AT 1 R), que induce vasoconstricción, antinatriuresis, secreción de aldosterona, activación del sistema nervioso simpático, no diferenciación y crecimiento celular, inflamación y daño a órganos diana, entre otras acciones perjudiciales (15).

La enzima convertidora de Angiotensina 2 (ECA2) es un homólogo de ECA y fue descubierto en 2000. A pesar de las similitudes entre ECA y ECA2, las funciones de estas dos enzimas son completamente diferentes (16). La actividad ECA2 del tejido es mayor que su actividad plasmática (17). ECA2 tiene una alta afinidad por Ang II y su eficiencia catalítica para Ang II es 
400 veces mayor que la Ang I. ECA2 puede mostrar diferencias en diferentes edades y sexos (18).

En la figura 1, se aprecia, la vía ECA2 - Ang II - AT1R se denomina eje SRA clásico, que desempeña un papel decisivo en la regulación, mientras que la vía basada en ECA2 - Ang 1-7 MasR se denomina eje SRA contrarregulador, que cumple un papel negativo en la regulación. Siendo función principal del eje SRA positivo es aumentar la tensión del sistema nervioso simpático, causar vasoconstricción, aumentar la presión arterial y promover la inflamación, la fibrosis y la hipertrofia miocárdica. El eje regulador negativo mediado por la ECA2 puede antagonizar estos efectos y se ha venido a denominar el "brazo protector", contrarrestando las acciones adversas de Ang II a través de AT 1 Rs (19)

Esta flexibilidad ayuda al cuerpo a responder de manera rápida y coordinada a estímulos específicos, desde todo el cuerpo hasta un área local, y juega un rol esencial en el mantenimiento de la homeostasis (16)

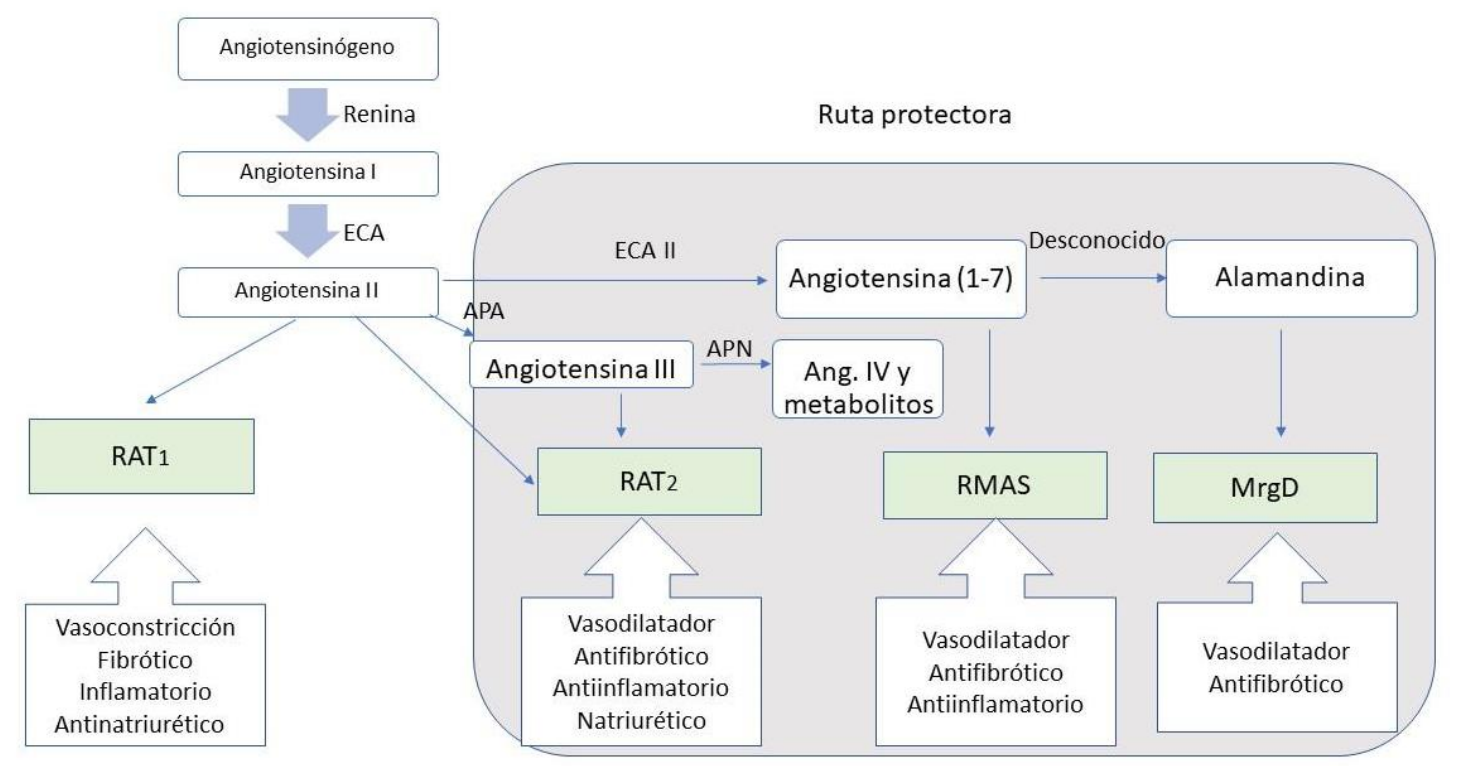

Figura 1. Sistema renina angiotensina

Los componentes del brazo protector incluyen ECA Il, angiotensina (1-7), vía del receptor Mas, la vía del receptor D acoplado a proteínas $\mathrm{G}(\mathrm{Mrg} \mathrm{D})$ de alamandina, Mas y la Angiotensina II, Angiotensina III, subtipo2 vía receptor ( $R A T_{2}$ ). Juntas, estas vías contrarreguladoras, funcionan en conjunto para reducir el im pacto perjudicial de la estimulación $\mathrm{R} A \mathrm{~T}_{1}$ inducida por Angiotensina II

ECA, enzima convertidora de angiotensina; APA, aminopeptidasa A; APN, aminopeptidasa N; RAT 1 , receptor de angiotensina tipo 1; RAT 2 , receptor de angiotensina tipo 2; $\mathrm{R}$ Mas , receptor Mas ; Mrg $D$, receptor acoplado a proteína G relacionado con Mas. Adaptado de Carey, 2017 (19) 


\section{ECA Y ECA2 EN LA GENÉTICA DEL POBLADOR DE ALTURA}

El gen ECA en 1990 fue descrito por Rigat, Hubert, Alhenc-Gelas et al., los mismos que detectaron la presencia (Inserción I) o ausencia (deleción D) de un fragmento de ADN de 250pb. Dentro del gen que representa el $47 \%$ de la variación fenotípica total del ECA sérica (20)

El polimorfismo del gen que codifica la ECA, con un alelo de "inserción" I en el intrón 16, en lugar de la ausencia o deleción (alelo D). El genotipo II que relacionado con una menor actividad de ECA en suero y tejido. Las dos versiones en el gen del alelo ECA, cuyas frecuencias (se han reportado como $0.24,0.50$ y 0.26 para los genotipos II, ID y DD respectivamente, frecuencia del alelo I, 0.49) (5) (21)

La delación del alelo (D) está asociado con mayor actividad en tejidos del ECA2 en el músculo esquelético. sugiriendo que el crecimiento muscular y el rendimiento físico. Sin embargo, el alelo (I) estaba asociado con rendimiento de resistencia mejorado como entre los alpinistas de gran altitud, levantamiento de pesas repetitivo, se mejora once veces en individuos homocigotos para el alelo de "inserción" II en comparación con aquellos homocigotos para el alelo de "deleción" DD. El alelo I también se ha encontrado en exceso con más frecuencia en corredores de distancia, remeros y otros atletas de élite (1)(22) (23) (24) (25) (26).

También hay un exceso del alelo I en quechuas de América del Sur que viven a más de 3000 m.s.n.m, en comparación con los caucásicos. La mayor frecuencia del alelo I podría haber facilitado la migración de quechuas ancestrales a las tierras altas (27). Esta sobre-representado comparado con los de caucásicos y una población de nativos americanos de las tierras bajas del Costa oeste de Canadá (28). El nivel reducido de ECA encontrado con el alelo I ha sido confirmado en europeos, Indios Pima y cohortes japonesas (29)(30)

El genotipo (II) está asociado con una vida media prolongada de bradiquinina produciendo un aumento en el flujo sanguíneo muscular, extracción de glucosa tasa y síntesis de proteínas en el músculo esquelético, (31)(32) y la producción reducida de angiotensina II (32)(33)(34)

Esto estaría asociada al mantenimiento de la saturación arterial de oxígeno $\left(\mathrm{SaO}_{2}\right)$ y sería ventajoso en $\mathrm{HH}$. También se encontró en escaladores de elite quienes tienen mayor $\mathrm{SaO}_{2}$ en reposo (88.8\% vs78\%) durante la hipoxia, en comparación con los controles (35)

El genotipo (DD) con Angiotensina II elevada (independiente de la presión barométrica) administrada a ratas da como resultado una reducción en la eficiencia metabólica y el desarrollo de caquexia. Así mismo está asociado con niveles elevados de ACE sérica, (36)

EI SRA podría desempeñar un papel en el mantenimiento de $\mathrm{SaO}_{2}$ en $\mathrm{HH}$ de varias maneras. La angiotensina II modula la respuesta vasoconstrictora pulmonar estimula el crecimiento 
hipertrófico e hiperplásico de los vasos sanguíneos, y células del músculo liso en la remodelación vascular pulmonar y regulación del impulso respiratorio hipóxico, a través de la modulación de la actividad quimiorreceptora del cuerpo carotídeo. Esto se atenúa por antagonismo del receptor AT1 y la inhibición de la ECA, pues ambos disminuyen el desarrollo en hipoxia crónica de hipertensión pulmonar y remodelación vascular (37)

La muscularización distal crónica inducida por hipoxia se asocia con aumentos tempranos y transitorios en los receptores AT2 y AT1, probablemente debido a hipoxia donde la respuesta vasotónica a angiotensina II se debe principalmente al subtipo AT1. Cuando se emplea el losartán antagonista de AT1 se inhibe completamente el efecto vasopresor de angiotensina II (38)(39).

Sin embargo, la selección no parece haber favorecido la transmisión transgeneracional del alelo de inserción de ACE en el quechua ya que la frecuencia de este alelo (0.777) no difieren de la población de Yanomami (Venezuela) a nivel del mar (0.847). Pero hay que considerar que el fondo genético también puede ser un factor donde la secuencia intrónica las frecuencias alélicas de ambos grupos de nativos americanos difieren significativamente de los caucásicos (Tabla 2)

Tabla 2. Genotipos y frecuencia de alelos para 287 bases de inserción(I)/ delación(D) y polimorfismo en el intrón 16 de la enzima de convertidora de angiotensina (ECA) en población quechua, Na-Dene y Caucásicos.

\begin{tabular}{|l|c|c|c|c|c|}
\hline & \multicolumn{3}{|c|}{ Genotipo } & \multicolumn{2}{c|}{ Frecuencia del alelo ECA } \\
\hline Población & $\mathrm{D} / \mathrm{D}$ & $\mathrm{I} / \mathrm{D}$ & $1 / \mathrm{I}$ & Deleción & Inserción \\
\hline Quechua & 2 & 24 & 37 & 0.223 & 0.777 \\
\hline Na-Dene & 5 & 16 & 29 & 0.260 & 0.740 \\
\hline Caucásicos & 9 & 21 & 4 & 0.574 & 0.426 \\
\hline
\end{tabular}

La frecuencia de los alelos en las poblaciones de los dos grupos de nativos americanos, difieren de los caucásicos $(p<0-05)$

Fuente: Adaptado de Rupert y col. (27)

Estos estudios pueden sugerir claramente que los habitantes de gran altitud, expres an niveles reducidos de ACE2 en sus pulmones (y otros tejidos). Por lo tanto, la adaptación al entorno de gran altitud podría hacer que los habitantes locales sean menos susceptibles a la penetración del virus SARS-CoV-2 y, en consecuencia, estén protegidos del desarrollo de la enfermedad que define el síndrome de dificultad respiratoria aguda (40).

\section{COVID19, ECA2 Y LESIÓN AGUDA DEL PULMÓN}

El tropismo viral en caso del COVID-19, es a los receptores ECA2, mediante la proteína S viral. La proteasa TMPRSS2 es principal en la entrada viral para la secuenciación de ARN unicelular (scRNAseq). Para el SARS, se descubrió que la afinidad de unión entre la proteína $S$ y el receptor 
ECA2 era determinante mayor determinante principal de las tasas de replicación viral y la gravedad de la enfermedad (41) (16)

El acoplamiento de la proteína espiga $S$ del coronavirus a ECA2 induce la extracción de ECA2 por el dominio metalopeptidasa ADAM 17 (pre factor de necrosis tumoral alfa ( TNF- $\alpha$ ) en la superficie celular y en las membranas del aparato de Golgi, con liberación de citocinas ancladas a membrana, moléculas de adhesión celular, receptores, ligandos y mayor captación celular de partículas de coronavirus. La actividad de ADAM17 está regulada por AT1R a través de los niveles de calcio intracelular y PMA, que induce su fosforilación a través de la actividad de PKC. TMPRSS2 también escinde ACE2 y se propuso que la escisión aumenta la entrada de SARScoronavirus (Figura 2) (42)(43)(44).

ECA2 y la proteasa TMPRSS2, se expresan en forma diferente en células epiteliales respiratorias e intestinales. Las células caliciformes / secretoras y células ciliadas nasales muestran la mayor expresión de ACE2 de todas las células epiteliales (45) otras proteasas como la catepsina B / L también pueden estar involucradas. (46)(47)(41)(45) Muchos genes asociados con la expresión epitelial ACE2 de las vías respiratorias están asociadas a la inmunidad innata, (46).

ECA2 es activo en la mayor parte de los tejidos y se distribuye ampliamente en el corazón, los riñones, los pulmones y los testículos en células epiteliales alveolares humanas y células epiteliales del intestino delgado, así como en células endoteliales arteriales y venosas y células de músculo liso arterial (16)

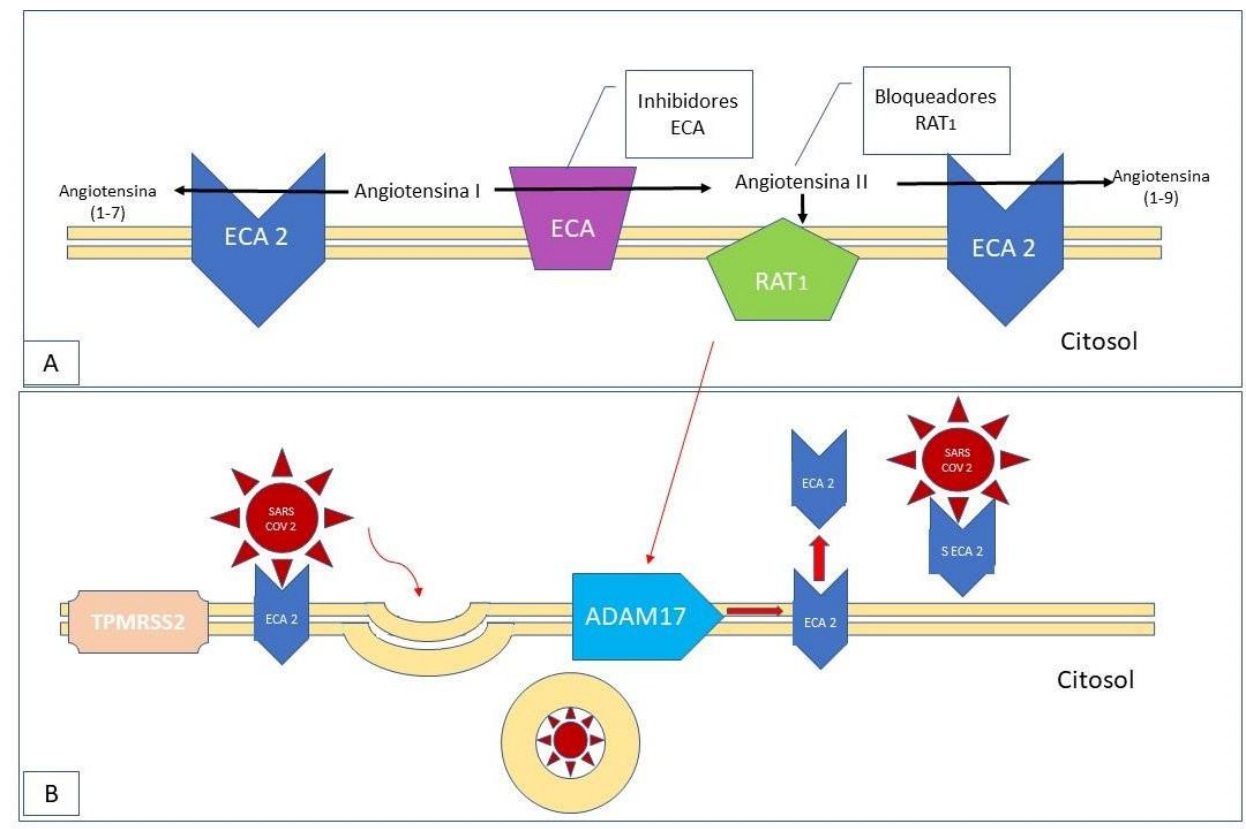

Figura 2. Relación entre ECA2 (enzima convertidora de angiotensina 2) y el SARS-COV2 
A. ECA2 convierte Angiotensina II en Angiotensina- (1-7) y Angiotensina I en Angiotensina- (1-9), pero no está bloqueada por los inhibidores de ACE, previenen la conversión de Angiotensina I a Angiotensina II.

B. ECA2 también se une e internaliza el SARS-Cov-2, después de cebar con la proteasa transmembrana, serina 2 (TMPRSS2). La eliminación de ECA2 unida a la membrana por la desintegrina ymetaloproteasa 17 (ADAM17) da como resultado la aparición de ECA2 soluble (s), que ya no puede mediar la entrada de SARS-Cov-2.

Ang II, a través de su receptor tipo 1 (RAT1) regula al alza ADAM17. Los bloqueadores RAT1 (ARB) evitarían esto. Adaptado de Danser y col., 2020. (44)

Se detectó ARN viral en las vías respiratorias superiores de pacientes sintomáticos con mayor viralidad o carga observadas en hisopos nasales en comparación con las obtenidas de la garganta La rápida propagación del SARS-CoV-2 sugiere una transmisión eficiente de persona a persona que parecería, a su vez, reemplazar las probabilidades de dependencia de las células epiteliales alveolares como punto primario de entrada y replicación viral $(46)(47)(41)(45)$. Sería muy importante para la infección inicial y puede servir como reservorio clave para propagación viral (3)

SARS COV2 son su proteína S se une a la ACE2 y reduce la disponibilidad de esta en el organismo, disminuyendo la acción biológica vasodilatadora de la angiotensina-1-7 (Ang 1-7) (4) Paralelamente se incrementa la actividad de la angiotensina II sobre el receptor AT1, produciendo la llamada tormenta de citoquinas, aumento de los factores inflamatorios, daño tisular pulmonar y desarrollo de injuria pulmonar (48). Así mismo, después del compromiso inicial de la proteína S de SARS-CoV-2, existe una baja regulación posterior de la abundancia de ACE2 en las superficies celulares. En modelos experimentales con ratones, la exposición a la proteína S del SARS-CoV-1 indujo una lesión pulmonar aguda, que está limitada por el bloqueo SRA (49). La infección viral continua y la replicación contribuyen a reducir la expresión de ECA2 en la membrana, al menos in vitro en células cultivadas (50)

Por lo antes mencionado, se propone utilizar bloqueadores disponibles del receptor 1 de angiotensina (AT1R), los ARA-II como losartán, como parte de la terapia para el COVID-19. (48).

\section{¿COMO SE COMPORTA EL COVID 19 EN LA ALTURA?}

Ante la presente pandemia recién empieza a observarse una correlación de la altura en zonas geográficas con los casos positivos y tasa de mortalidad por COVID- 19 (40)(51)(52). Igualmente, en el estudio realizado por Xu y col. Se observa que a mayor altura menores casos positivos de COVID19, para lo cual se correlaciono en tiempo real la pandemia y con un modelo de elevación digital en relación con la altitud (51).

En el estudio realizado por Arias y col., se encuentra relación de la altura con los casos positivos de COVID19 es decir a mayor altura menos casos (véase figura 3), (40). Se encontró una clara disminución cuando la población afectada vive a una altitud de más de 3.000 msnm (40).

El estudio de países como el protectorado del Tíbet (China), Bolivia o Ecuador o la correlación de las tasas de mortalidad en ciudades con más de 100,000 habitantes a más de 2500 msnm a 
nivel global, incluyen en estas observaciones la disminución de casos de casos de fallecimientos (52). Si bien podrían existir posibles sesgos como el tiempo de presencia de enfermedad, el sub registro en el diagnóstico de infectados por la falta de pruebas diagnósticas o deficiencia en los sistemas de notificación y la implementación de medidas de aislamiento social en forma temprana que deberían ser estudiadas a profundidad, sin embargo muchas de estas medidas se vienen estandarizando a finales de abril (40) Asumiendo como el más sensible la tasa de mortalidad por COVID -19 frente a la tasa de letalidad. Hallando diferencia significativa de la tasa de mortalidad de la región en relación al global del país respectivo (52). Algunos autores tratan de explicar esta relación por los cambios drásticos en la temperatura entre la noche y el día, la sequedad del aire y los altos niveles de radiación de luz ultravioleta (UV) la menor densidad del aire y la mayor distancia entre las moléculas a gran altitud, el tamaño del inóculo de virus en el aire debe ser menor que a nivel del mar (40). La radiación de luz ultravioleta A (UVA) y B (UVB) que es capaz alterar los enlaces moleculares del ADN y el ARN, por lo que a gran altitud puede actuar como un desinfectante natural el SARS-CoV-2, deberían acortar la vida media del virus (53)

Sin embargo, se conoce que en regiones de gran altitud existen otras afecciones virales como influenza que no se ven influenciados por la altura y muestran junto con otras virosis respiratorias, como la primera causa de morbimortalidad en áreas de altura (54). Así también se toma en cuenta que la aclimatación fisiológica a gran altitud está asociada con una mayor ventilación, transporte de oxígeno arterial aumentado y una mayor oxigenación de los tejidos principalmente mediada por la eritropoyetina y podría ser terapia potencial al SDRA por COVID19 , hechos que aun estarían en propuesta pues serian complementarios al principal factor para la adaptación de la altura y afección del COVID-19 (40)

En nuestra evaluación coincidimos con la de Arias Reyes en la que se afirma una correlación positiva entre la tasa de infección de SARS-CoV-1 y ACE2 en las células epiteliales pulmonares. Es importante destacar que tanto el SARS-CoV-1 como el SARS-CoV-2 se unen a ACE2 (40)(55) 


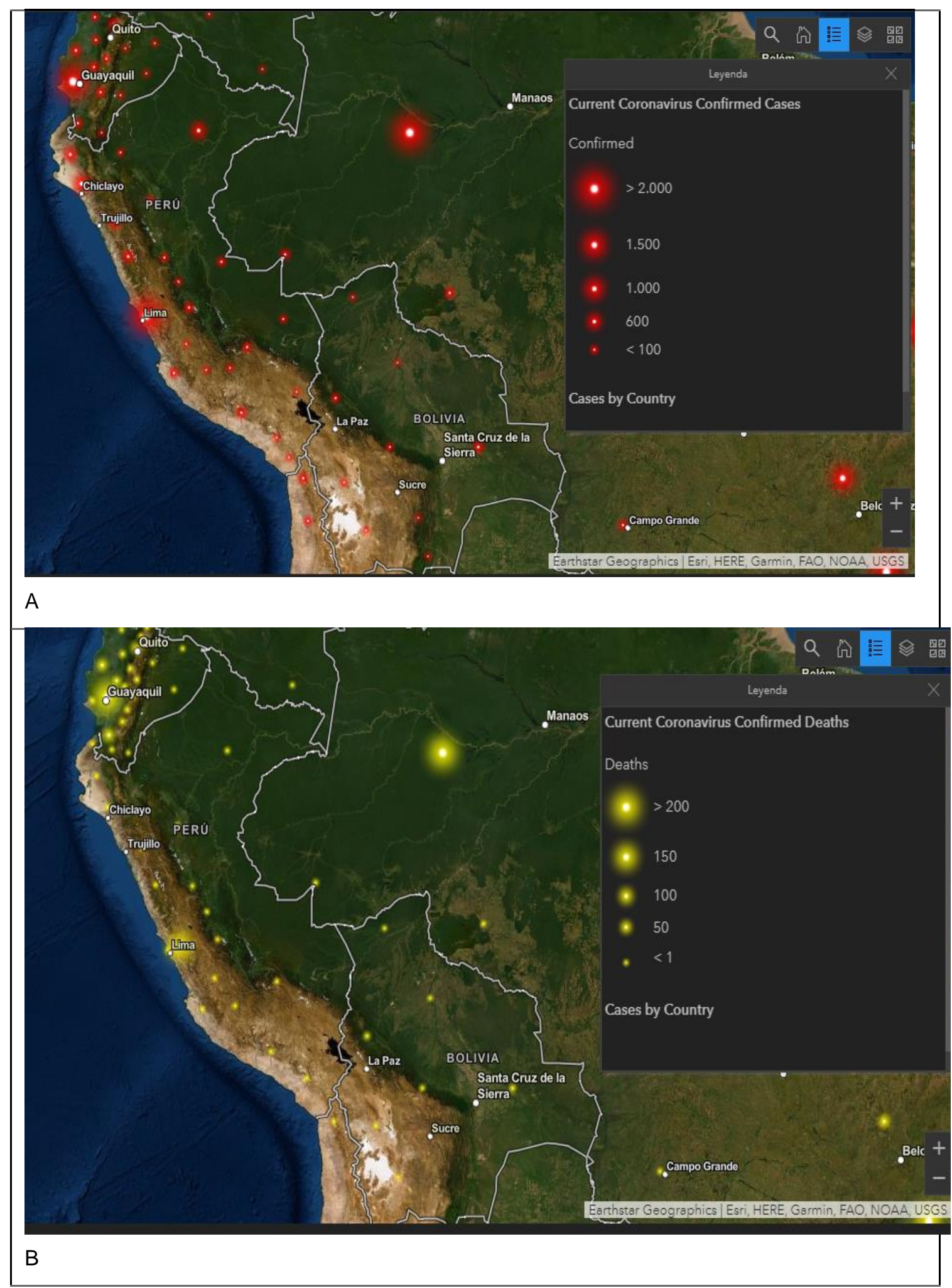




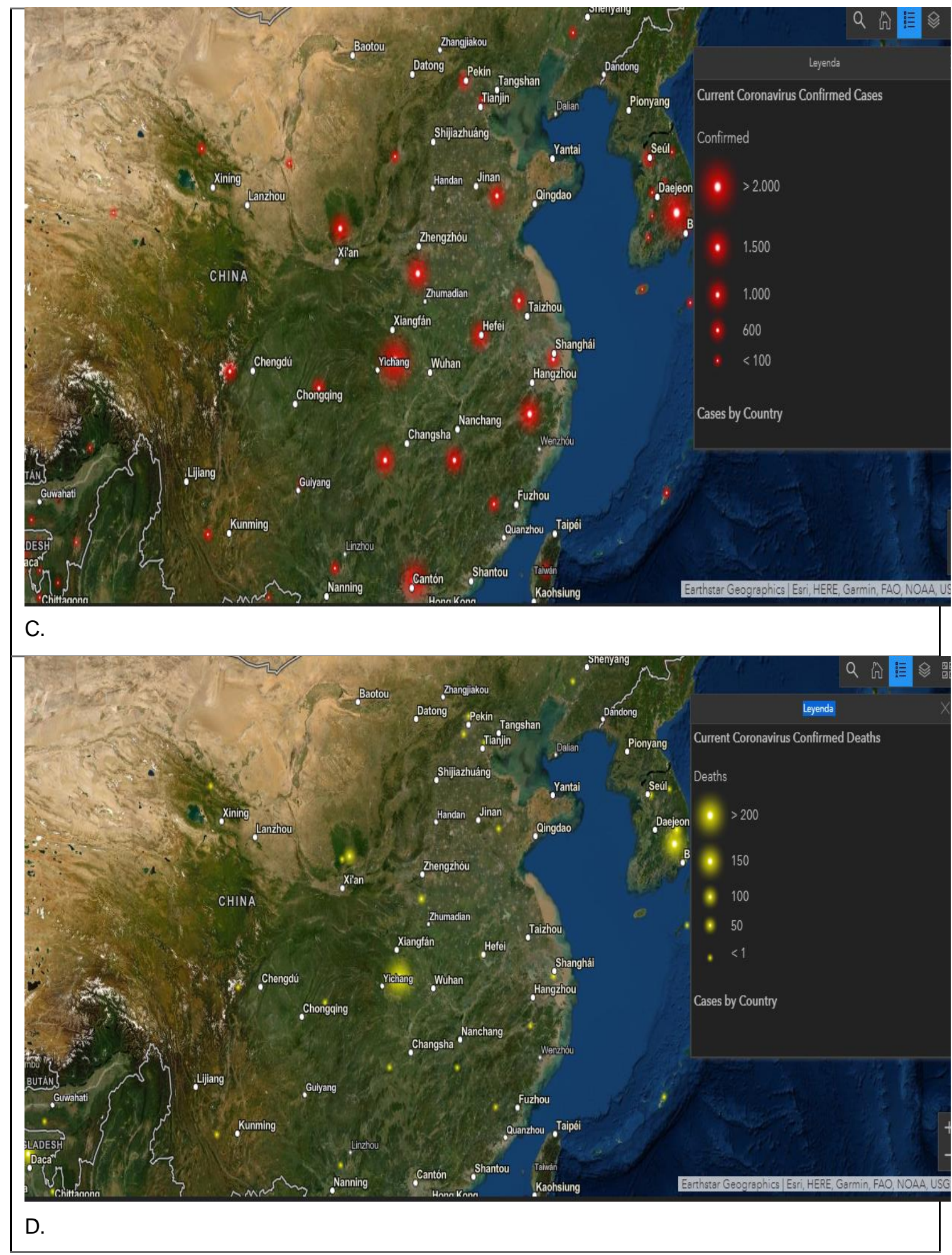

Figura 3. Distribución geográfica y altitudinal de la pandemia de COVID-19 tres países de Sur América (Bolivia, Perú y Ecuador) y China. Los casos confirmados puntos rojos y casos fatales puntos amarillos. Al 1 de mayo 2020.

https://early-

alert.maps.arcgis.com/apps/opsdashboard/index.html\#/20bfbf89c8e74c0494c90b1ae0fa7b78

A: Casos confirmados COVID-19 en tres países de Sudamérica (Bolivia, Perú, Ecuador) 1/Mayo/2020

B: Casos fatales COVID-19 en tres países de Sudamérica (Bolivia, Perú, Ecuador) 1/Mayo/2020 

C: Casos confirmados COVID-19 China 1/Mayo/2020
D: Casos fatales COVID-19 China 1/Mayo/2020

\section{COMENTARIOS FINALES. -}

Proponemos en el presente artículo que el comportamiento de la pandemia del SARS COV-19 es diferente según las zonas geográficas a nivel global. Se observa un comportamiento diferente en los pobladores que habitan a más de $2500 \mathrm{msnm}$., frente a los que habitan a menor altitud, esto se puede concluir de la comparación de la presencia de casos, mortalidad en regiones con poblaciones mayores de 100000 habitantes frente al reto de la pandemia. Lo cual es significativamente diferente, sin considerar otras medidas de contención profilaxis que haya 0 estén desarrollando para frenar la pandemia.

Por lo tanto, la disminución de la expresión de ACE2 en endotelios pulmonares en elevada altitud habitante podría representar una protección fisiológica para el edema pulmonar severo y letal.

Cuando se evalúan los posibles factores de estas diferencias por la altura, se observa la fisiopatología de la infección por COVID -19, la cual se basa en la acción de la angiotensina II, que tiene un efecto deletéreo por la liberación que se produce luego del ingreso del genoma del virus. Cuyo ingreso implica la unión de la proteína $S$ viral con el enzima convertidores de angiotensina II, que se encuentra en la superficie de múltiples células en especial del aparato respiratorio.

Por las características del hombre de altura muy extensamente estudiadas, en su adaptación crónica y las diferentes características que adopta ya de tiempo ancestral, dentro de sus sistemas de adaptación ha desarrollado una adaptación del SRA. Donde la disminución de angiotensina II, renina y la poca presencia de la ECA2 dentro de otras modificaciones, inhiben la expresión del recetor a la proteína $S$ o espícula del SARS COV2 desarrollando poca capacidad de infección y virulencia.

Estas apreciaciones son importantes, puesto que las complicaciones de esta patología serían más benignas en altura, lo que no exime que la presencia y patogenia del virus sea menor. Dependiendo exclusivamente del hospedero intermediario (habitante de altura)

Quedan muchas interrogantes en este punto como es: comprobar la presencia y severidad del cuadro relacionado con la cantidad de angiotensina II, ECA 2, en pacientes infectados de altura mayor y menor de $2500 \mathrm{msnm}$. Evaluar la presencia de los alelos de inserción I y $\mathrm{D}$ en las dos poblaciones (en alta y baja altura) y su correlación con la severidad de la enfermedad, independientemente del sitio de residencia, de forma que pueda se pueda identificar pacientes con mayor riesgo de cuadros graves. Así como estimular la activación del Factor Inducible por 
Hipoxia 1 (HIF-1) para personas que la tienen reprimida a nivel de alturas menores de 2500 msnm para una readaptación represión de la presencia de ACE2, de forma que mejoren su capacidad de respuesta al COVID19

De comprobarse esta teoría, las medidas de aislamiento o cuarentenas tendrían que ser replanteadas, para diferentes grupos poblacionales respecto a la altura, conociendo las concentraciones de angiotensina II, ECA en sangre y de alelos I o D para ECA2.

\section{REFERENCIAS BIBLIOGRÁFICAS.}

1. Avellanas Chavala ML. A journey between high altitude hypoxia and critical patient hypoxia: What can it teach us about compression and the management of critical disease? Med Intensiva (English Ed [Internet]. 2018 Aug 1 [cited 2020 Apr 25];42(6):380-90. Available from:

https://www.sciencedirect.com/science/article/abs/pii/S217357271830119X

2. Kiely D. Haemodynamic and endocrine effects of type 1 angiotensin II receptor blockade in patients with hypoxaemic cor pulmonale. Cardiovasc Res [Internet]. 1997 Jan;33(1):201-8. Available from: https://academic.oup.com/cardiovascres/articlelookup/doi/10.1016/S0008-6363(96)00180-0

3. Zhang H, Penninger JM, Li Y, Zhong N, Slutsky AS. Angiotensin-converting enzyme 2 (ACE2) as a SARS-CoV-2 receptor: molecular mechanisms and potential therapeutic target. Intensive Care Med [Internet]. 2020 Apr 3;46(4):586-90. Available from: http://link.springer.com/10.1007/s00134-020-05985-9

4. Kuster GM, Pfister O, Burkard T, Zhou Q, Twerenbold R, Haaf P, et al. SARS-CoV2: should inhibitors of the renin-angiotensin system be withdrawn in patients with COVID19? Eur Heart J [Internet]. 2020 Mar 20; Available from: https://academic.oup.com/eurheartj/advancearticle/doi/10.1093/eurheartj/ehaa235/5810479

5. Simmons G, Zmora P, Gierer S, Heurich A, Pöhlmann S. Proteolytic activation of the SARS-coronavirus spike protein: Cutting enzymes at the cutting edge of antiviral research. Antiviral Res [Internet]. 2013 Dec;100(3):605-14. Available from: https://linkinghub.elsevier.com/retrieve/pii/S016635421300288X

6. Pereira-Victorio CJ, Huamanquispe-Quintana J, Castelo-Tamayo LE. Revista Peruana de Medicina Experimental y Salud Publica. Instituto Nacional de Salud; 2014 [cited 2020 Apr 26]. 473-479 p. Available from:

http://www.scielo.org.pe/scielo.php?script=sci_arttext\&pid=S1726 46342014000300010\&lng=es\&nrm=iso\&tlng=es

7. Mishra A, Mohammad G, Norboo T, Newman JH, Qadar Pasha MA. Lungs at highaltitude: Genomic insights into hypoxic responses. J Appl Physiol [Internet]. 2015;119(1):1-15. Available from: https://www.ncbi.nlm.nih.gov/pubmed/25911686

8. Ward M, Milledge J, West J. High Altitude Medicine And Physiology. Phyladelphia: 
University of Pennsylvania; 1989. 2-7 p.

9. Krasney JA. A neurogenic basis for acute altitude illness. Med Sci Sports Exerc [Internet]. 1994;26(2):195-208. Available from: https://doi.org/10.1249/00005768199402000-00010

10. Belén M, De La Cruz J, Garcia L, Santiango A. Influencia de la altitud sobre la regulaciónde la presión arterial en el ejercicio físico efectos sobre el sistema reninaangiotensina-aldosterona Archivos de medicina del deporte. Arch Med del Deport [Internet]. 2004;21:287-2965. Available from:

http://archivosdemedicinadeldeporte.com/articulos/upload/Original_influencia_287_102.p df

11. Cano I, Mickael M, Gomez-Cabrero D, Tegnér J, Roca J, Wagner PD. Importance of mitochondrial $\mathrm{P}(\mathrm{O} 2)$ in maximal $\mathrm{O} 2$ transport and utilization: a theoretical analysis. Respir Physiol Neurobiol [Internet]. 2013 Dec;189(3):477-83. Available from: https://linkinghub.elsevier.com/retrieve/pii/S1569904813003029

12. Zhang R, Wu Y, Zhao M, Liu C, Zhou L, Shen S, et al. Role of HIF-1a in the regulation ACE and ACE2 expression in hypoxic human pulmonary artery smooth muscle cells. Am J Physiol - Lung Cell Mol Physiol [Internet]. 2009;297(4):631-40. Available from: https://www.ncbi.nlm.nih.gov/pubmed/19592460

13. Semenza GL. HIF-1: Mediator of physiological and pathophysiological responses to hypoxia. J Appl Physiol [Internet]. 2000;88(4):1474-80. Available from: https://journals.physiology.org/doi/pdf/10.1152/jappl.2000. 88.4.1474

14. Prabhakar NR, Semenza GL. Adaptive and maladaptive cardiorespiratory responses to continuous and intermittent hypoxia mediated by hypoxia-inducible factors 1 and 2 . Physiol Rev [Internet]. 2012;92(3):967-1003. Available from: https://www.ncbi.nlm.nih.gov/pubmed/22811423

15. Carey RM, Siragy HM. Newly recognized components of the renin-angiotensin system: Potential roles in cardiovascular and renal regulation. Endocr Rev [Internet]. 2003;24(3):261-71. Available from: https://www.ncbi.nlm.nih.gov/pubmed/12788798

16. Cheng $\mathrm{H}$, Wang $\mathrm{Y}$, Wang $\mathrm{G}-\mathrm{Q}$. Organ-protective effect of angiotensin-converting enzyme 2 and its effect on the prognosis of COVID-19. J Med Virol [Internet]. 2020;(March):1-5. Available from: https://www.ncbi.nlm.nih.gov/pubmed/32221983

17. Donoghue M, Hsieh F, Baronas E, Godbout K, Gosselin M, Stagliano N, et al. A Novel Angiotensin-Converting Enzyme-Related Carboxypeptidase (ACE2) Converts Angiotensin I to Angiotensin 1-9. Circ Res [Internet]. 2000 Sep 1 [cited 2020 Apr 27];87(5). Available from: https://www.ahajournals.org/doi/10.1161/01.RES.87.5.e1

18. Xudong $X$, Junzhu C, Xingxiang W, Furong Z, Yanrong L. Age- and gender-related difference of ACE2 expression in rat lung. Life Sci [Internet]. 2006 Apr 4 [cited 2020 Apr 27];78(19):2166-71. Available from: https://www.sciencedirect.com/science/article/pii/S0024320505010386

19. Carey RM. AT2 receptors: Potential therapeutic targets for hypertension. Am J Hypertens [Internet]. 2017;30(4):339-47. Available from: 
https://academic.oup.com/ajh/article/30/4/339/2623060

20. Rigat B, Hubert C, Alhenc-Gelas F, Cambien F, Corvol P, Soubrier F. An insertion/deletion polymorphism in the angiotensin I-converting enzyme gene accounting for half the variance of serum enzyme levels. J Clin Invest [Internet]. 1990;86(4):1343-6. Available from: https://www.ncbi.nlm.nih.gov/pubmed/1976655

21. Miller GJ, Bauer KA, Barzegar S, Cooper JA, Rosenberg RD. Increased Activation of the Haemostatic System in Men at High Risk of Fatal Coronary Heart Disease. Thromb Haemost [Internet]. 1996;75(05):767-71. Available from:

https://www.ncbi.nlm.nih.gov/pubmed/8725721

22. Montgomery HE, Marshall R, Hemingway H, Myerson S, Clarkson P, Dollery C, et al. Human gene for physical performance. Nature [Internet]. 1998;393(6682):221-2. Available from: https://www.ncbi.nlm.nih.gov/pubmed/9607758

23. Pasha MAQ, Khan AP, Kumar R, Grover SK, Ram RB, Norboo T, et al. Angiotensin converting enzyme insertion allele in relation to high altitude adaptation. Ann Hum Genet [Internet]. 2001 [cited 2020 Apr 26];65(6):531-6. Available from: https://www.cambridge.org/core/product/154E6AF9D11B52EF5D710BA69FB0FE0D

24. Woods DR, Pollard AJ, Collier DJ, Jamshidi Y, Vassiliou V, Hawe E, et al. Insertion/deletion polymorphism of the angiotensin I-converting enzyme gene and arterial oxygen saturation at high altitude. Am J Respir Crit Care Med [Internet]. 2002;166(3):362-6. Available from: https://www.ncbi.nlm.nih.gov/pubmed/12153971

25. Myerson S, Hemingway H, Budget R, Martin J, Humphries S, Montgomery H. Human angiotensin I-converting enzyme gene and endurance performance. J Appl Physiol [Internet]. 1999;87(4):1313-6. Available from: https://journals.physiology.org/doi/full/10.1152/jappl.1999.87.4.1313

26. Montgomery H, Clarkson P, Barnard M, Bell J, Brynes A, Hajnal J, et al. Angiotensinconverting-enzyme gene insertion/deletion polymorphism and response to physical training. Lancet [Internet]. 1999 Feb 13 [cited 2020 Apr 26];353(9152):541-5. Available from: https://www.sciencedirect.com/science/article/pii/S0140673698071311

27. Rupert JL, Devine D V., Monsalve M V., Hochachka PW. Angiotensin-converting enzyme (ACE) alleles in the Quechua, a high altitude South American native population. Ann Hum Biol [Internet]. 1999;26(4):375-80. Available from: https://www.tandfonline.com/doi/abs/10.1080/030144699282688

28. Salzanoa F, Callegari-Jacques SM. South American Indians: a case study in evolution. Oxford: Clarendon Press; 1988. 5 p.

29. Kohno M, Yokokawa K, Minami M, Kano H, Yasunari K, Hanehira T, et al. Association between angiotensin-converting enzyme gene polymorphisms and regression of left ventricular hypertrophy in patients treated with angiotensin-converting enzyme inhibitors. Am J Med [Internet]. 1999 May 1 [cited 2020 Apr 26];106(5):544-9. Available from: https://www.sciencedirect.com/science/article/abs/pii/S0002934399000674

30. Foy CA, McCormack LJ, Knowler WC, Barrett JH, Catto A, Grant PJ. The angiotensin-I converting enzyme (ACE) gene I/D polymorphism and ACE levels in Pima Indians. J 
Med Genet [Internet]. 1996;33(4):336-7. Available from:

https://jmg.bmj.com/content/33/4/336.short

31. Brink M, Wellen J, Delafontaine P. Angiotensin II causes weight loss and decreases circulating insulin-like growth factor I in rats through a pressor-independent mechanism. J Clin Invest [Internet]. 1996;97(11):2509-16. Available from:

https://www.jci.org/articles/view/118698

32. Murphey LJ, Gainer J V., Vaughan DE, Brown NJ. Angiotensin-converting enzyme insertion/deletion polymorphism modulates the human in vivo metabolism of bradykinin. Circulation [Internet]. 2000;102(8):829-32. Available from: https://www.ahajournals.org/doi/full/10.1161/01.CIR.102.8.829

33. Hagberg JM, Ferrell RE, Mccole SD, Wilund KR, Moore GE. VO(2max) is associated with ACE genotype in postmenopausal women. J Appl Physiol [Internet]. 1998;85(5):1842-6. Available from: https://journals.physiology.org/doi/full/10.1152/jappl.1998.85.5.1842

34. Brown NJ, Blais C, Gandhi SK, Adam A. ACE Insertion/Deletion Genotype Affects Bradykinin Metabolism. J Cardiovasc Pharmacol [Internet]. 1998;32(3):373-7. Available from:

https://journals.Iww.com/cardiovascularpharm/Fulltext/1998/09000/ACE_Insertion_Deleti on_Genotype_Affects.6.aspx

35. Oelz O, Howald H, Di Prampero PE, Hoppeler H, Claassen H, Jenni R, et al. Physiological profile of world-class high-altitude climbers. J Appl Physiol [Internet]. 1986 May 1;60(5):1734-42. Available from: https://www.physiology.org/doi/10.1152/jappl.1986.60.5.1734

36. Butler R, Morris AD, Struthers AD. Angiotensin-Converting Enzyme Gene Polymorphism and Cardiovascular Disease. Clin Sci [Internet]. 1997 Nov 1;93(5):391-400. Available from: https://portlandpress.com/clinsci/article/93/5/391/76891/AngiotensinConvertingEnzyme-Gene-Polymorphism-and

37. Cargill RI, Lipworth BJ. Lisinopril Attenuates Acute Hypoxic Pulmonary Vasoconstriction in Humans. Chest [Internet]. 1996 Feb 1 [cited 2020 May 3];109(2):424-9. Available from: https://www.sciencedirect.com/science/article/abs/pii/S0012369215456715

38. Chassagne C, Eddahibi S, Adamy C, Rideau D, Marotte F, Dubois-Randé JL, et al. Modulation of angiotensin II receptor expression during development and regression of hypoxic pulmonary hypertension. Am J Respir Cell Mol Biol [Internet]. 2000;22(3):32332. Available from: http://www.atsjournals.org/doi/abs/10.1165/ajrcmb.22.3.3701

39. Meyrick BO, Perkett EA. The Sequence of Cellular and Hemodynamic Changes of Chronic Pulmonary Hypertension Induced by Hypoxia and Other Stimuli. Am Rev Respir Dis [Internet]. 1989 Nov, 140(5):1486-9. Available from: http://www.atsjournals.org/doi/abs/10.1164/ajrccm/140.5.1486

40. Arias-Reyes C, Zubieta-DeUrioste N, Poma-Machicao L, Aliaga-Raduan F, CarvajalRodriguez F, Dutschmann M, et al. Does the pathogenesis of SARS-CoV-2 virus decrease at high-altitude? Respir Physiol Neurobiol [Internet]. 2020 Jun;277:103443. 
Available from: https://linkinghub.elsevier.com/retrieve/pii/S1569904820301014

41. Hoffmann M, Kleine-Weber H, Krüger N, Müller M, Drosten C, Pöhlmann S. The novel coronavirus 2019 (2019-nCoV) uses the SARS-coronavirus receptor ACE2 and the cellular protease TMPRSS2 for entry into target cells. bioRxiv [Internet]. 2020 Jan 1;2020.01.31.929042. Available from:

http://biorxiv.org/content/early/2020/01/31/2020.01.31.929042.abstract

42. Black RA, Rauch CT, Kozlosky CJ, Peschon JJ, Slack JL, Wolfson MF, et al. A metalloproteinase disintegrin that releases tumour-necrosis factor- $\alpha$ from cells. Nature [Internet]. 1997 Feb;385(6618):729-33. Available from:

http://www.nature.com/articles/385729a0

43. Moss ML, Jin S-LC, Milla ME, Burkhart W, Carter HL, Chen W-J, et al. Cloning of a disintegrin metalloproteinase that processes precursor tumour-necrosis factor- $\alpha$. Nature [Internet]. 1997 Feb;385(6618):733-6. Available from:

http://www.nature.com/articles/385733a0

44. Danser AHJ, Epstein M, Batlle D. Renin-Angiotensin System Blockers and the COVID19 Pandemic. Hypertension [Internet]. 2020 Jun;75(6):1382-5. Available from: https://www. ahajournals.org/doi/10.1161/HYPERTENSIONAHA.120.15082

45. Li W, Zhang C, Sui J, Kuhn JH, Moore MJ, Luo S, et al. Receptor and viral determinants of SARS-coronavirus adaptation to human ACE2. EMBO J [Internet]. $2005 \mathrm{Apr}$ 20;24(8):1634-43. Available from: http://emboj.embopress.org/cgi/doi/10.1038/sj.emboj.7600640

46. Sungnak W, Huang N, Bécavin C, Berg M, Network HLB. SARS-CoV-2 Entry Genes Are Most Highly Expressed in Nasal Goblet and Ciliated Cells within Human Airways. 2020 Mar 13 [cited 2020 Apr 27]; Available from: https://arxiv.org/abs/2003.06122

47. Matsuyama S, Nagata N, Shirato K, Kawase M, Takeda M, Taguchi F. Efficient Activation of the Severe Acute Respiratory Syndrome Coronavirus Spike Protein by the Transmembrane Protease TMPRSS2. J Virol [Internet]. 2010 Dec 15;84(24):12658 LP 12664. Available from: http://jvi.asm.org/content/84/24/12658.abstract

48. Gurwitz D. Angiotensin receptor blockers as tentative SARS-CoV-2 therapeutics. Drug Dev Res [Internet]. 2020 Mar 4;ddr.21656. Available from: https://onlinelibrary. wiley.com/doi/abs/10.1002/ddr.21656

49. Kuba K, Imai Y, Rao S, Gao H, Guo F, Guan B, et al. A crucial role of angiotensin converting enzyme 2 (ACE2) in SARS coronavirus-induced lung injury. Nat Med [Internet]. 2005 Aug 10;11(8):875-9. Available from:

http://www.nature.com/articles/nm1267

50. Dijkman R, Jebbink MF, Deijs M, Milewska A, Pyrc K, Buelow E, et al. Replicationdependent downregulation of cellular angiotensin-converting enzyme 2 protein expression by human coronavirus NL63. J Gen Virol [Internet]. 2012 Sep 1;93(9):19249. Available from: https://www.microbiologyresearch.org/content/journal/jgv/10.1099/vir.0.043919-0

51. Xu B, Gutierrez B, Mekaru S, Sewalk K, Goodwin L, Loskill A, et al. Epidemiological data 
from the COVID-19 outbreak, real-time case information. Sci Data [Internet]. 2020 Dec 24;7(1):106. Available from: http://www.nature.com/articles/s41597-020-0448-0

52. Cardona A, Montoya M. COVID19 en población residente de zonas geográficas a alturas superiores a $2500 \mathrm{msnm}$. Situa UNSAAC [Internet]. 2020; Available from: http://revistas.unsaac.edu.pe/index.php/SITUA/article/view/204

53. Andrade M. La radiación ultravioleta en tiempos de cuarentena. 2020; Available from: https://www.paginasiete.bo/opinion/2020/4/10/la-radiacion-ultravioleta-en-tiempos-decuarentena-252240.html

54. Hodinka RL. Respiratory RNA Viruses. Microbiol Spectr [Internet]. 2016 Aug 18;4(4). Available from:

http://www.asmscience.org/content/journal/microbiolspec/10.1128/microbiolspec.DMIH2 0028-2016

55. Lu R, Zhao X, Li J, Niu P, Yang B, Wu H, et al. Genomic characterisation and epidemiology of 2019 novel coronavirus: implications for virus origins and receptor binding. Lancet [Internet]. 2020 Feb;395(10224):565-74. Available from: https://linkinghub.elsevier.com/retrieve/pii/S0140673620302518

SITUA. 2020; 23(2): 1-19

DOI: https://doi.org/10.51343/si.v23i2.310 